stellar force dominates by a factor $\sim 10^{2}-$ $10^{3}$, and hence the self gravity of the disk is negligible. Therefore it is invalid to use the Bertin and Mark theory.

A more appropriate approximation would be to consider the bending modes of a gas disk due to motion in the stellar potential alone. The modes obtained from such an analysis ${ }^{8}$ which are most closely analogous to the bending wave of Bertin and Mark have a frequency of $\sim\left(4 \pi G \rho_{*}\right)^{1 / 2}$ yielding a very small group velocity in comparison with the phase velocity.

A. H. NELSON
Department of Applied Mathematics
and Astronomy,
University College,
Cardiff CF1 1XL, UK

1. Blitz, L., Mark, J.W.-K. \& Sinha, R. P. Nature 290, $120-121(1980)$.

2. Bertin, G. \& Mark, J.W.-K. Astr. Astrophys. 88, 289-297 (1980).

3. Hunter, C. \& Toomre, A. Astrophys. J. 155, 747-776 (1969).

4. Maihara, T., Naoki, O., Sugiyama, T. \& Okuda, H. Publ. astr. Soc. Jap. 30, 1-19 (1978).

5. Mihalas, D. \& Routly, P. M. Galactic Astronomy 196 Freeman, San Francisco, 1968)

6. Oort, J. H. A. Rev. Astr. Astrophys. 15, 295-362 (1977).

7. Gordon, M. A. \& Burton, W. B. Astrophys. J. 208, 346$353(1976)$

8. Nelson, A. H. Mon. Not. R. astr. Soc. 177, 265-274 (1976),

BLITZ AND MARK REPLY-Nelson's suggestion that the Bertin and Mark ${ }^{1}$ bending wave theory is inapplicable to the dynamics of the gaseous disk at the galactic centre seems to be based on an incomplete analysis of the singular perturbation problem involved when a disk of small (but not altogether negligible) mass is immersed in a larger spheroidal component.

For disk mass much smaller than spheroidal masses, it is not appropriate to omit the disk mass terms. The 'kinematic waves' resulting from this omission (see equation (D7) of (ref. 1) dominated by $\nu_{z}^{2}$ on the right-hand side) dissipates on the rather short differential rotation shearing time scale $|\mathrm{d} \Omega / \mathrm{d} \ln r|^{-1}$. On the other hand, including the dynamical effects of the small disk mass results in self-gravitating waves with the much longer group propagation dissipation time, $\sim 2 R d k / d \omega$, which has the dispersion relation (D7) of (ref. 1). The neglect of similar terms in Nelson's ${ }^{2}$ formalism for modes symmetric about the galactic plane would fail to produce the familiar Jeans instability (the bending waves are the antisymmetric disturbances about the galactic plane). By contrast, Bertin and Mark have included terms of the 'sound wave' type included by Nelson, but within the context of galaxy warps, stellar dispersive speeds squared are much larger than the corresponding terms in the gas.

In any case, Bertin and Mark have included terms which reflect more completely the dynamics of the disk. The conclusion with or without these additional terms is that the scaling law $\left(r \Sigma^{2} h \sim\right.$ constant) used by us is consistent with the observations of the inner disk of the
Galaxy as well as the warps of the outer region of several galaxies as shown by Lake and Mark ${ }^{3}$.

JAMES W.-K. MARK LEO BLITZ

Astronomy Department,

University of California,

Berkeley, California 94720, USA

1. Bertin, G. \& Mark, J.W.-K. Astr. Astrophys. 88, 289-297 (1980).

2. Nelson, A. H. Mon. Not. R. astr. Soc. 177, 265-274 (1976).

3. Lake, G. \& Mark, J.W.-K. Nature 287, 705-706 (1980).

\section{Lipoprotein assembly in Xenopus yolk-platelet crystals}

FROM his determination of the space group of yolk-platelet crystals from several new species, Lange ${ }^{1}$ has questioned earlier data of ours for similar lipoprotein crystals from Xenopus ${ }^{2}$. The new data are based entirely on electron micrographs from fixed sectioned specimens, a procedure we intentionally avoided during our studies. This evidence as well as a few other statements made in the paper need to be scrutinized.

The first data shown (Fig. 1 of ref. 1) are three 'tracings' of electron diffraction patterns from yolk crystals of the species Triturus. It is unfortunate that Lange did not publish the patterns themselves because frequently the high noise level from such poorly diffracting specimens makes interpretation of the patterns difficult. Furthermore, a more analytical description including the averages and standard deviations of the observed lattice spacings would have been useful. It is, after all, the unit cell spacings which we and now Lange, have used to identify specific projections ${ }^{1,3}$.

In further criticism of the data presentation, the space group determination using Figs 2 and 3 of ref. 1 is confusing because no optical diffraction patterns are shown and the micrographs were obtained from two different species. Lange's Fig. 3, for example, bears a strong resemblance to the hol zone from lipoprotein crystals of $X_{\text {Xenopus }}{ }^{3}$. Lange describes this as the $h k o$ zone, suggesting that simple relabelling of the $b$ and $c$ axes would make the results consistent with the earlier work ${ }^{3}$. Indeed, we agree that the projection shown in Fig. 3 should have $p g g$ symmetry. As the $b$ and $c$ axes in the unit cell for Xenopus are roughly the same length, they may equally well be similar in other species and could easily be interchanged. Unit cell dimensions can be precisely obtained for wet and dry crystals by using $\mathrm{X}$-ray powder methods ${ }^{3}$.

In image reconstruction studies, the best way to determine the symmetry of a given projection is to examine the phases of strong symmetry-related reflections ${ }^{4}$. In the studies of the lipoprotein complex from Xenopus, phases of each centric projection were calculated and were then shifted to minimize the difference between that calculated and the nearest real value $e^{2,5}$. Two pairs of strong reflections on the 001 projection $(1,3,0$ and $1, \overline{3}, 0 ; 2,3,0$ and $2, \overline{3}, 0$ ) had phase relationships consistent with only pmg symmetry ${ }^{5}$. Six pairs of reflections had phase relationships consistent with pgg symmetry for the view we assigned to the hol projection. Many of the projections used in the image reconstruction were studied earlier by optical filtering methods. Even on such filtered images, both the number of molecules and the symmetry can be difficult to ascertain ${ }^{6}$.

Furthermore we take issue with Lange's discussion of the reliability of positive versus negative staining ${ }^{1}$ in special reference to our image reconstruction results. Certainly the appearance of lattice micrographs will be different when one is positively stained and the other negatively stained. However, the immobilization of stain (positive staining effects) adjacent to solvent channels filled with stain (negative staining), is unlikely to produce major artefactual results. Thus, we emphasize the importance of negative stain as well as the lack of chemical fixation in the earlier study $^{2}$.

Finally, we note that although the work on Xenopus was severely limited by the chance finding of different crystal fragments, it still contained data from 10 different views selected from several hundred electron micrographs ${ }^{2,3}$.

LEONARD J. BANASZAK

Department of Biological Chemistry,

Washington University,

School of Medicine,

St Louis, Missouri 63110, USA

DOUGLAS H. OHLENDORF

Institute of Molecular Biology,

University of Oregon,

Eugen, Oregon 97403, USA

1. Lange, R. H. Nature 289, 329-330 (1981).

2. Ohlendorf, D. H., Wrenn, R. F. \& Banaszak, L. J. Nature 272, 28-32 (1978).

3. Ohlendorf, D. H., Collins, M. L., Puronen, E. D. Banaszak, L. J. \& Harrison, S. C. J. molec. Biol. 99, 153-165 (1975).

4. Unwin, P. N. T. J. molec. Biol. 98, 235-242 (1975)

5. Ohlendorf, D. H. thesis, Washington Univ. (1978)

6. Ohlendorf, D. H., Wrenn, R. F., Collins, M. L. \& Banaszak, L. J. in Cellular Function and Molecula Structure (eds Agris, P. \& Sykes, B.) 159-164 (Academic, New York, 1978)

LANGE REPLIES-If the symmetric lipoprotein aggregates in yolk platelets of higher anamniotes are orthorhombic crystals as reported for Xenopus laevis ${ }^{1}$, their space group is $\mathrm{P} 22_{1} 2_{1} 2_{1}$, as documented by symmetry pgg of their axial projections $^{2}$ at usual electron microscope resolution in eight different species above the cyclostome level (among them $X$. laevis).

\section{Institute for Anatomy}

R. H. LANGE

and Cytobiology,

Aulweg 123,

D-6300 Giessen, FRG

1. Ohlendorf, D. H., Wrenn, R. F. \& Banaszak, L. J. Nature
272, 28-32 (1978).

2. Lange, R. H. Nature $289,329-330$ (1981). 